\title{
Produção de biomassa e de óleo essencial de três quimiotipos (I, II e III) e quatro acessos de erva-cidreira [Lippia alba (Mill.) N. E. Brown] no sul do Estado do Tocantins
}

\section{Biomass yield and essential oil from three chemotypes (I, II and III) and four accessions of lemongrass [Lippia alba (Mill.) N. E. Brown] in the southern State of Tocantins}

\author{
João Josué Batista Neto ${ }^{1 *}$, Tarcisio Castro de Barros Leal ${ }^{1}$, Melquezedeque do Vale Nunes ${ }^{1}$, \\ Henrique Guilhon de Castro ${ }^{1}$, Suetônio Fernandes dos $\operatorname{Santos}^{1}$ e Raimundo Wagner de \\ Souza Aguiar ${ }^{1}$
}

${ }^{l}$ Departamento de Agronomia; Universidade Federal do Tocantins; 77402-970; Gurupi - TO - Brasil.

\begin{abstract}
This study aimed to evaluate the production of essential oil from three chemotypes for experiment 1 and four accessions of L. alba from different municipalities in southern Tocantins for experiment 2, with these three repetitions, the design of randomized blocks. The essential oil was obtained via hydrodistillation in Clevenger apparatus. Were evaluated: dry weight, content and yield of essential oil per plant. In experiment 1 there was no statistically significant difference in any of the variables analyzed. In the second experiment, there was significant difference between treatments in Gurupi / Cariri in relation to access to Figueirópolis / Alvorada with regard to oil content, which did not differ on the characteristics analyzed for dry mass, when analyzed if the yield per plant was a significant difference between the fits of Gurupi and Cariri in relation to access to Figueirópolis and Alvorada.

Key-words: Lippia alba, hits, chemotypes, essential oil
\end{abstract}

\section{INTRODUÇÃO}

Desde as primeiras civilizações, as plantas com propriedades medicinais são utilizadas como fitoterápicos. A simples observação dos recursos naturais com propriedades terapêuticas deu início aos estudos e aplicações mais efetivas para a população, principalmente a partir do século XVIII, com o surgimento de ciências agregadas. Nos anos mais recentes, tem-se observado o aumento acentuado do uso dessas plantas pela população em todo o mundo (Lorenzi e Matos, 2002), e, segundo Camargo (2001), a tendência é de contínuo crescimento. $O$ setor fitoterápico movimenta cerca de um bilhão de reais por ano, sendo que no Brasil, o comércio de fitomedicamentos correspondeu a $6 \%$ do faturamento total de medicamentos e envolveu $\mathrm{R} \$$ 400 milhões, só em 2004 (De Le Cruz, 2005). Apesar de as estatísticas divulgadas serem pouco descritivas e imprecisas para o comércio referente a plantas medicinais, ainda é possível notar, com clareza, a importância desses produtos para o Brasil, principalmente quando com valor agregado.

A erva-cidreira [Lippia alba (Mill.) N. E. Brown] é uma das plantas de real importância farmacológica, sendo utilizada em diversos programas de fitoterapia. A espécie é largamente utilizada no Brasil devido às suas propriedades calmante, espasmolítica suave, analgésica, sedativa, ansiolítica e levemente expectorante (Mattos et al., 2007). As folhas são utilizadas pela

Author for correspondence: rwsa@uft.edu.br 
população na forma de infuso, tintura, banhos, cataplasmas e inalação. Sua aplicação inclui tratamento de desordens gastrointestinais, doenças respiratórias (pela atividade antibacteriana), problemas hepáticos e gastrite (Pascual et al., 2001a), e intoxicações em geral.

A composição química dos óleos essenciais é determinada por fatores genéticos, porém, outros fatores podem acarretar alterações significativas na produção dos metabólitos secundários. De fato, os metabólitos secundários representam uma interface química entre as plantas e o ambiente. Os estímulos decorrentes do ambiente, no qual a planta se encontra, podem redirecionar a rota metabólica, ocasionando a biossíntese de diferentes compostos (Bezerra et al., 2008).

A composição do óleo essencial de L. alba apresenta variação quantitativa e qualitativa, levando à separação em quimiotipos (Matos, 1996; Frigheto et al., 1998; Zoghbi et al., 1998), os quais poderiam apresentar atividades farmacológicas distintas, bem como diferenças morfológicas (Matos, 1996; Correa, 1992). As variações na composição do óleo essencial e características morfológicas têm sido observadas dependendo da origem geográfica do material, o que levou a hipótese de que seriam consequência da influencia de fatores ambientais (Retamar, 1994; Zoghbi et al., 1998).

É de grande importância que se estabeleçam linhas de ação voltadas para o desenvolvimento de técnicas de manejo ou cultivo (pesquisas fitotécnicas) das plantas com potencial terapêutico, considerando-se a sua utilização pelo homem e a manutenção do equilíbrio dos ecossistemas (Mattos, 2007). É fundamental que estas técnicas sejam desenvolvidas respeitando-se as condições edafoclimáticas regionais, uma vez que a produção de princípios ativos pelas plantas pode ser intensamente afetada pelo ambiente de cultivo (Zoghbi et al., 1998).

Desta forma, objetivou-se, com este trabalho, a avaliação de três quimiotipos (I, II e III) e quatro acessos de Lippia alba, provenientes de quatro municípios do sul do estado do Tocantins, tendo como parâmetro de análise o teor de óleo essencial, a produção de biomassa e o rendimento do óleo por planta.

\section{MATERIAL E MÉTODOS}

Os experimentos foram conduzidos na Estação Experimental de Pesquisa (EEP), da Universidade
Federal do Tocantins, Campus de Gurupi, localizada a $11^{\circ} 43^{\prime} \mathrm{S}$ e $49^{\circ} 04^{\prime} \mathrm{N}$, a $278 \mathrm{~m}$ de altitude. O clima é do tipo B1wA' úmido com moderada deficiência hídrica, segundo a classificação climática de Köppen (1948). A temperatura média anual é de $26{ }^{\circ} \mathrm{C}$, variando de $22{ }^{\circ} \mathrm{C}$ a $32{ }^{\circ} \mathrm{C}$. A umidade relativa média do ar é de $76 \%$ e chove, em média, $1804 \mathrm{~mm}$ anualmente.

$\mathrm{O}$ solo da área experimental é da classe latossolo vermelho amarelo eutrófico, com textura média, cuja análise química e física é apresentada na Tabela 1. Os dados de precipitação pluviométrica e temperatura são apresentados no Quadro 1 e os dados de radiação solar são apresentados na Figura 1.

Para se avaliar a produção do óleo essencial de três quimiotipos e de quatro acessos de Lippia alba no sul do estado do Tocantins foram realizados dois experimentos de campo, no ano de 2011. O delineamento experimental de ambos os experimentos foi o de blocos ao acaso, com três repetições, com parcelas medindo $3 \mathrm{~m} \times 2 \mathrm{~m}$ e espaçamento entre plantas de $0,5 \mathrm{~m} \times 0,5 \mathrm{~m}$, contendo quatro linhas de seis plantas, totalizando vinte quatro plantas por parcela. Em cada parcela, foram consideradas como plantas úteis as quatro plantas centrais das duas linhas centrais, sendo todas as outras plantas consideradas bordadura. $\mathrm{Na}$ preparação das mudas, evitou-se retirar estacas de plantas doentes, com ataque de pragas e danos mecânicos. Foram retiradas, das plantas matrizes, estacas com $20 \mathrm{~cm}$ de comprimento e três gemas, e diâmetro superior a $0,5 \mathrm{~cm}$. As mesmas foram colocadas para enraizar em bandejas de isopor com substrato comercial Plantimax - R, por aproximadamente 30 dias, antes de serem plantadas definitivamente no campo. As capinas foram realizadas manualmente com enxada de acordo com as necessidades e durante o período de estiagem foi programado irrigação por aspersão com turno de rega de três dias, utilizando-se equipamento do tipo auto-propelido de barras. Não houve necessidade de controle fitossanitário por não ter havido incidência de doenças, assim como a prática de adubação foi dispensada em virtude do residual de culturas anteriores na área do experimento, conforme dados de análise de solo. O plantio no campo foi realizado em três de fevereiro na avaliação dos três quimiotipos e 26 de Março na avaliação dos quatro acessos. A idade padrão de colheita em ambos os experimentos foi aos 90 dias após o plantio a campo. 
Tabela 01. Análise química do solo.

\begin{tabular}{|c|c|c|c|c|c|c|c|c|c|c|c|}
\hline $\begin{array}{l}\text { Profundidade } \\
\mathrm{cm}\end{array}$ & $\begin{array}{c}\mathrm{pH} \\
\mathrm{H}_{2} \mathrm{O}\end{array}$ & $\begin{array}{l}\mathrm{P} \\
\mathrm{mg} \mathrm{dm}\end{array}$ & ${ }_{-3} \mathrm{~K}$ & $\mathrm{Al}^{3+}$ & $\mathrm{H}+\mathrm{Al}$ & $\begin{array}{c}\mathrm{Ca}^{2+} \\
\mathrm{mol}_{\mathrm{c}} \mathrm{dm}^{-3}\end{array}$ & $\mathrm{Mg}^{2+}$ & SB & $\mathrm{T}$ & $\begin{array}{l}\mathrm{V} \\
\%\end{array}$ & $\begin{array}{l}\mathrm{MO} \\
\mathrm{g} \mathrm{dm}\end{array}$ \\
\hline $\begin{array}{l}0-20 \\
\text { Textura }\end{array}$ & 5,2 & $\begin{array}{c}4,2 \\
\text { Argila } \\
250 \mathrm{~g} / \mathrm{kg}\end{array}$ & 4,9 & $\begin{array}{c}0,0 \\
\text { Silte } \\
120 \mathrm{~g} / \mathrm{kg}\end{array}$ & 3,2 & $\begin{array}{c}2,3 \\
\text { Areia } \\
630 \mathrm{~g} / \mathrm{kg}\end{array}$ & $\begin{array}{l}1,0 \\
\mathrm{~g}\end{array}$ & 3,3 & 6,5 & 50,7 & 25,2 \\
\hline
\end{tabular}

Fonte: Laboratório de análise de Solos da UFT - Campus de Gurupi.

\begin{tabular}{|c|c|c|c|}
\hline \multicolumn{4}{|c|}{$\begin{array}{l}\text { Quadro 1. Dados de precipitação pluviométrica e } \\
\text { temperatura do ano de } 2011 \text { em Gurupi - TO. }\end{array}$} \\
\hline Mês & 2011 & Mínima & Máxima \\
\hline Jan & 243,2 & 21,6 & 31,0 \\
\hline $\mathrm{Fev}$ & 233,6 & 21,9 & 31,4 \\
\hline Mar & 259,8 & 22,4 & 31,4 \\
\hline Abr & 114,0 & 21,9 & 32,1 \\
\hline Mai & 97,8 & 20,6 & 32,7 \\
\hline Jun & 8,2 & 17,7 & 32,8 \\
\hline Jul & 0,0 & 17,6 & 33,5 \\
\hline Ago & 0,0 & 18,3 & 34,9 \\
\hline Set & 3,0 & 20,5 & 35,4 \\
\hline Out & 190,4 & 21,9 & 33,5 \\
\hline Nov & 204,6 & 21,8 & 31,9 \\
\hline Dez & 206,1 & 21,7 & 31,1 \\
\hline
\end{tabular}

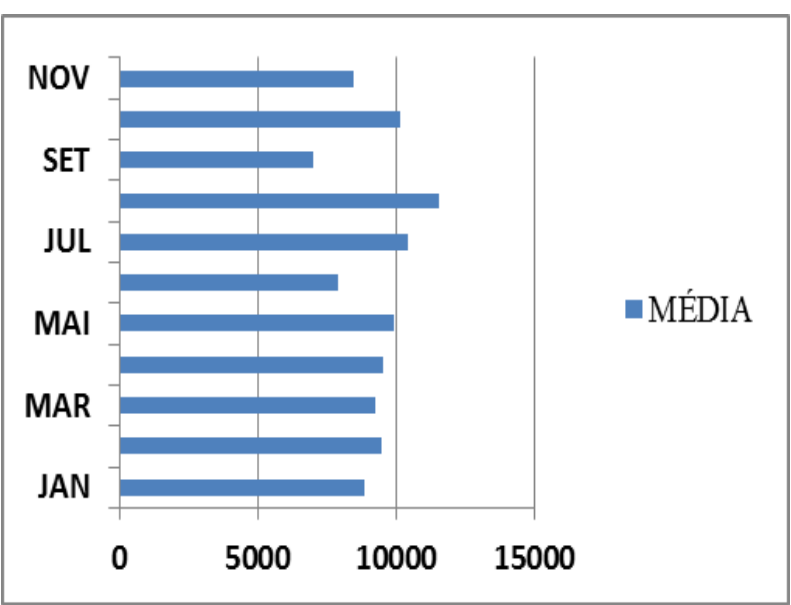

Figura 1. Média da radiação solar anual em Gurupi TO.

Fonte: Estação Climatológica da UFT campus de Gurupi.
Para a obtenção da massa fresca e seca foram realizadas colheitas nos horários entre 8 e 9 horas da manhã e o material foi seco à sombra por $48 \mathrm{~h}$ no Laboratório de Ecofisiologia Vegetal da UFT.

A extração do óleo essencial foi realizada por hidrodestilação em aparelho tipo Clevenger, utilizando $50 \mathrm{~g}$ de folhas desidratadas. Estas foram colocadas em um balão volumétrico de $500 \mathrm{~mL}$, preenchendo o volume com água destilada $\mathrm{e}$ submetida à ebulição por período de $1 \mathrm{~h}$, usando a mesma metodologia descrita em Radünz et al. (2002).

As variáveis analisadas foram: massa seca das folhas (em gramas), rendimento de óleo por planta (em $\mathrm{mL}$ ) e o teor de óleo essencial (\%) tanto para os quimiotipos, quanto para os acessos de Lippia alba (erva-cidreira).

Os resultados foram submetidos à análise de variância e os contrastes de médias comparados pelo teste de Tukey a 5\% de significância.

\section{RESULTADOS E DISCUSSÃO}

De acordo com a Tabela 2, verifica-se que não houve diferença significativa quanto ao teor, à massa seca das folhas e o rendimento de óleo por planta, entre os três quimiotipos.

Segundo Guimarães et al. (2008), a composição quantitativa e qualitativa dos óleos essenciais de Lippia alba é determinada por fatores genéticos. Porém, outros fatores podem acarretar alterações significativas na produção dos metabólitos secundários. As variações na composição do óleo essencial e características morfológicas têm sido observadas dependendo da origem geográfica do material, o que levou à hipótese de que seria conseqüência da influência de fatores ambientais (Retamar, 1994; Zoghbi et al.,1998).

Tavares et al. (2005), analisando o óleo essencial de folhas de três quimiotipos de Lippia alba (Mill.) N. E. Br. (Verbenaceae) cultivados em condições semelhantes observaram que as diferenças na composição e quantidade do óleo essencial dos três quimiotipos não constituem produto da influência de fatores ambientais, mas 
refletem variação genotípica entre as plantas já que tais diferenças mantiveram-se em plantas cultivadas lado a lado em um mesmo canteiro. Verificaram, também, que a extração de óleo essencial de L. alba para a obtenção de citral carvona e linalol deve ser efetuada em plantas em fase de crescimento vegetativo quando $o$ rendimento do óleo e os teores dos componentes majoritários são maiores.

As informações encontradas e confirmadas pelos autores acima, reforçam os resultados obtidos quanto às características estudadas para os três quimiotipos em Gurupi-TO.

Tabela 2. Teor (\%), rendimento de óleo essencial (mL/planta) e massa seca das folhas $(\mathrm{g})$, dos três quimiotipos de Lippia alba, no município de Gurupi - TO.

\begin{tabular}{lccc}
\hline Quimiotip/Caract. & Teor (\%) & Rendimento/Planta $(\mathbf{m L})$ & Massa Seca das Folhas/Parcela (g) \\
\hline Quimiotipo I & $1,92 \mathrm{a}$ & $0,34 \mathrm{a}$ & $147,11 \mathrm{a}$ \\
Quimiotipo Ii & $2,00 \mathrm{a}$ & $0,45 \mathrm{a}$ & $164,49 \mathrm{a}$ \\
Quimiotipo Iii & $2,00 \mathrm{a}$ & $0,34 \mathrm{a}$ & $140,23 \mathrm{a}$ \\
Média & 1,97 & 0,38 & 150,61 \\
\hline
\end{tabular}

$\mathrm{CV}($ Teor $)=12.15 \% ; \mathrm{CV}$ (Rendimento $)=21.46 \% ; \mathrm{CV}$ (Massa Seca de Folhas $)=12.01 \%$; Médias seguidas de mesma letra na linha não diferem estatisticamente entre si, de acordo com o teste de Tukey a $5 \%$ de probabilidade.

Tabela 3. Teor (\%), Massa seca das folhas (g) e rendimento de óleo essencial (mL/planta) de acessos de Lippia alba procedentes de quatro municípios do sul do Estado do Tocantins, cultivados em Gurupi - TO.

\begin{tabular}{lccc}
\hline Acessos/Caract. & Teor & Rendimento/Planta $(\mathbf{m L})$ & Massa Seca das Folhas/Parcela $(\mathbf{g})$ \\
\hline Gurupi & $2,26 \mathrm{a}$ & $0.27 \mathrm{a}$ & $97.89 \mathrm{a}$ \\
Cariri & $2,00 \mathrm{a}$ & $0.26 \mathrm{a}$ & $110.02 \mathrm{a}$ \\
Figueirópolis & $0,92 \mathrm{~b}$ & $0.11 \mathrm{~b}$ & $95.14 \mathrm{a}$ \\
Alvorada & $1,00 \mathrm{~b}$ & $0.11 \mathrm{~b}$ & $89.25 \mathrm{a}$ \\
Média & 1,54 & 0.19 & 0,98 \\
\hline CV $($ Teor $)=12.72 \% ; C V$ (Rendimento) $=15.33 \% ; \mathrm{CV}($ Massa Seca de Folhas) $=10.53 \% ;$ Médias seguidas da \\
mesma letra na linha não diferem estatisticamente entre si, de acordo com o teste Tukey a 5\% de probabilidade.
\end{tabular}

De acordo com a tabela 3 acima, os acessos de $L$. alba provenientes de Gurupi e Cariri não diferiram entre si e foram superiores aos acessos de Figueirópolis e Alvorada para as características analisadas de teor e rendimento de óleo por planta, os quais, por sua vez, não diferiram entre si. Quanto à massa seca de folhas, não houve diferença significativa entre os quatro acessos de L. alba avaliados.

Camêlo et al. (2011), avaliando o potencial agronômico de diferentes acessos de Lippia alba provenientes de regiões distintas (Bahia, Rio de Janeiro e Ceará), contataram diferenças significativas na produção de óleo essencial.

A interferência dessas variações no fenótipo da planta é classicamente representada pela localidade de cultivo, genótipos e anos diferentes, assim como pela interação entre os três fatores (Borém e Miranda, 2005).

Assim, variações do meio ambiente, principalmente aquelas diferentes do habitat natural, conduzem a modificações nos indivíduos quanto aos perfis de composição dos metabólitos secundários (Retamar, 1994; Zoghbi et al., 1998) de forma muito dinâmica, sendo estes responsáveis pelas relações entre o indivíduo e o ambiente onde ele se encontra. Estas afirmações destes autores, reforçam o resultado apresentado neste experimento.

\section{RESUMO}

Este estudo teve como objetivo avaliar a produção de óleo essencial de três quimiotipos de Lippia alba (experimento 1) e quatro acessos de plantas desta espécie provenientes de diferentes municípios do sul do Tocantins (experimento 2). O delineamento utilizado foi o de blocos ao acaso, com três repetições. O óleo essencial foi obtido via hidrodestilação em aparelho Clevenger. Foram avaliadas: massa seca de folhas, teor e rendimento de óleo essencial por planta. No experimento 1 não houve diferença estatística significativa em nenhuma das variáveis analisadas. Já no experimento 2, houve diferença significativa nos 
acessos de Gurupi e Cariri em relação aos acessos de Figueirópolis e Alvorada no que diz respeito ao teor e rendimento de óleo por planta, os quais não diferiram entre si quanto à massa seca de folhas.

Palavras-chave: Lippia alba, acessos, quimiotipos, óleo essencial

\section{REFERÊNCIAS}

Bezerra, A. M. E.; Medeiros-Filho, S; Oliveira, L. D. M.; Silveira, E. R. (2008), Produção e composição química da macela em função da época de colheita. Horticultura Brasileira, 26: 2629.

Borém, A. e Miranda, G.V. (2005), Melhoramento de plantas (eds). Universiadade Federal de Viçosa, 4. Ed, 85-111.

Camargo, R. C. R. Biologia floral da Lippia alba (Verbenaceae), atividade antibacteriana $\mathrm{e}$ caracterização de amostras de méis de Apis mellifera L.. Tese (Doutorado em Zootecnia Nutrição e Produção Animal) - Universidade Estadual Paulista Júlio de Mesquita Filho, Botucatu, 2001.

Carvalho filho, J. L. S. Horário de colheita, temperatura e tempo de secagem no óleo essencial de manjericão (Ocimum basilicum L.). Monografia (Graduação em Engenharia Agronômica) Universidade Federal de Sergipe, São Cristóvão, 2004.

Cartilha da Oficina apresentada na Feira do Empreendedor no estande do GOVERNO DO ESTADO DO MATO GROSSO, 2005.

Correa C. B. V. (1992), Contribuição ao estudo de Lippia Alba (Mill.) N. E. Br. ex Britt \& Wilson erva-cidreira. Revista Brasileira de Farmacognosia, 73, 57-64.

De La Cruz, M G. (2011), Frutos, Ervas e Temperos: $\mathrm{O}$ remédio na sua cozinha e no seu quintal - Camêlo L. C. A., Blank A. F., Ehlert, C. R. D. Caracterização morfológica e agronômica de acessos de ervacidreira- brasileira [Lippia alba (Mill.) N. E. Br.]. V. 7, N. 5., Scientia Plena, São Paulo - Brasil.

Embrapa. (2006), Centro Nacional e Pesquisa em Solos. Sistema Brasileiro de Classificação de
Solos. Brasília: Embrapa - SPI; Rio de Janeiro: Embrapa - Solos, 306p.

Gobbo-Neto, L.; Lopes, N. P. (2007), Plantas medicinais: fatores de influencia no conteúdo de metabolitos secundários. Química Nova, 30, 374381.

Guimarães, L. G. L; Cardoso, M. G. C; Zacaroni, L. M; Lima, R. K; Pimentel, F. A; Morais, A. R. (2008), Influência da luz e da temperatura sobre a oxidação do óleo essencial de capim-limão (Cymbopogon citratus (D.C.) STAPF). Química Nova, 31, 1476-1480.

Frighetto, N.; Oliveira, J. G.; Siani, A. C.; Chagas, K. C. (1998), Lippia alba Mill N. E. Br. (Verbenaceae) as a source of linalool. Journal of Essential Oil Research, 10, 578-580.

Lorenzi, H. e Matos, F. J. A. (2004), Plantas medicinais no Brasil: Nativas e exóticas cultivadas. Nova Odessa, SP: Instituto Plantarum, $512 \mathrm{p}$.

Köppen, W. (1948), Climatologia: Fondo de Cultura Económica. México.

Matos, F. J. de A. (1996), As ervas-cidreiras do nordeste do Brasil - Estudo de três quimiotipos de Lippia alba (Mil.) N.E.Brown (Verbenaceae). Farmagnosia. Revista Brasileira de Farmagnosia, 77, 65-67.

Matos, F. J. A. (1996), As ervas-cidreira do Nordeste do Brasil - Estudo de três quimiotipos de Lippia alba (Mill.) N. E. Brown (Verbenaceae) Revista Brasileira de Farmacologia, 77, 65-67.

Mattos, S. H.; Innecco, R.; Marco, C. A.; Araújo, A. V. (2007), Plantas medicinais e aromáticas cultivadas no Ceará: tecnologia de produção e óleos essenciais. Fortaleza: Banco do Nordeste do Brasil, (série BNB - ciência e tecnologia 2), 61-63.

Mattos, S. H. (2007), Perspectivas do cultivo de plantas medicinais para a fitoterapia no Estado do Ceará. Horticultura Brasileira, 18, 45-46.

Nascimento, I. B.; Innecco, R.; Marco, C. A.; Mattos, S. H.; Nagao, E. O. (2003), Efeito do horário de corte no óleo essencial de capim-santo. Revista Ciência Agronômica, 34, 169-172. 
Pascual, M. E.; Slowing, K.; Carretero, E.; Mata, D. S.; Villar, A. (2001), Lippia: Traditional uses, chemistry and pharmacology: a review. Journal of Ethnopharmacology, 76, 201-214.

Radün, L. L.; Melo, E. C.; Berbert, P. A.; Barbosa, L. C. A.; Rocha, R. P.; Grandi, A. M. (2002), Efeitos da temperatura do ar de secagem sobre a qualidade do óleo essencial de alecrim pimenta (Lippia sidoides cham). Revista Brasileira de Armazenamento, 27, 9-13.

Retamar, J. A. (1994), Variaciones fi toquimicas de la especie Lippia alba (salvia morada) y sus aplicaciones en la química fina. Essenze Derivati Agrumari, 16, 55-60.

Ricciardi, G. A. L., Ricciardi, A. I. A. (2000), Efecto de lãs variciones estacionales sobre $\mathrm{La}$ composicion química del aceite esencial de plantas de "Salvia morada" de Sáenz Peña (Chaco).
Silva, F.; Santos, R. H. S.; Diniz, E. R.; Barbosa, L. C. A.; Casali, V. W. D.; Lima, R. R. (2003), Teor e composição do óleo essencial de manjericão (Ocimum basilicum L.) em dois horários e duas épocas de colheita. Revista Brasileira de Plantas Medicinais, 6, 33-38.

Tavares E. S.; Julião L. S.; Lopes D.; Bizzo H. R. (2005), Análise do óleo essencial de folhas de três quimiotipos de Lippia alba (Mill.) N. E. Br. (Verbenaceae) cultivados em condições semelhantes. Revista Brasileira de Farmacognosia, 15, 1-5.

Zoghbi, M. G. B.; Andrade, E. H. A.; Santos, A. S.; Silva, N. H. L.; Maia, J. G. S. (1998), Essential oils of Lippia alba (Mill.) N.E.Br. growing wild in the brazilian amazon. Flavour and Fragrance Journal, 13, 47-48. 